\title{
Analysis heat transfer in engineering barriers used FEM and experimental method
}

\author{
Dariusz Andrzejewski ${ }^{1, *}$, and Mariusz Prazmowski ${ }^{1}$ \\ ${ }^{1}$ Opole University of Technology, Faculty of Mechanical Engineering, 5 Mikolajczyka St., Opole, \\ Poland
}

\begin{abstract}
Purpose of the calculations is to determine the temperature field at various points in the area in question. Estimate the losses of heat given off by the analysed structures to the environment. Numerical algorithm based on the Finite Element Method for steady flow of heat and areas of heterogeneous. To analyse was used windows with double-glazed unit made PCV with different option gasses and glasses. Verification the simulation which used numerical procedures by experimental method.
\end{abstract}

\section{Introduction}

To analysis we have an engineering barrier (windows panel set double glazed resized specially for experimental check in heater chamber with different glasses and used gases) which will be realised algorithm numerical simulation. Besides checking the optimally distances for provide the best insulation between double glazed set has been proven impact on the size panel set for thermal insulation. Specify the temperature field in the distinguished points of the area in question arising from the way the geometrical discretization of the continuum to estimate the losses of heat given off by the design of this analysed. To solve a issues was applicable finite element method (FEM) in variant published in the[1].To finally calculation was used author program to automatic generation net differential where finally equations was develop iterative method Gauss-Seidel $[2,3]$.

We try find optimum distance between two set of glasses in window panel for smallest heat transfer coefficient, use correct glass, gasses and material for construction and design in order to improve save energy used author program and compare to commercial program "Fluent". As well opportunity to use the internal heating plates of glass to eliminate thermal bridge causing condensation of the water at the bottom of the glass. Opportunity to use specific system of cooling the glass to blocked move the heat from the outside to the inside.

\section{Material and methods}

\subsection{Mathematics process}

In mathematics problem it's described differential equations [2-4]:

${ }^{*}$ Corresponding author: d.andrzejewski@po.opole.pl 


$$
\frac{\partial}{\partial x_{j}}\left[\lambda_{j}\left(\frac{\partial T_{j}}{\partial x_{j}}\right)\right]=0 \mathrm{e}=1,2, \ldots, E
$$

$\lambda_{\mathrm{e}}$-thermal conductivity

$\mathrm{T}_{\mathrm{e}}$-temperature field in area

For testing calculation checked effective value for numerical calculation. Any convection in "empty" area made effective coefficient instead real thermal conductivity.

Equations for calculation:

$$
\frac{\partial}{\partial \mathrm{x}_{1}}\left[\lambda_{\mathrm{e}}\left(\frac{\partial \mathrm{T}_{\mathrm{e}}}{\partial \mathrm{x}_{1}}\right)\right]+\frac{\partial}{\partial \mathrm{x}_{2}}\left[\lambda_{\mathrm{e}}\left(\frac{\partial \mathrm{T}_{\mathrm{e}}}{\partial \mathrm{x}_{2}}\right)\right]=0
$$

Adopted boundary conditions Robin:

$$
\mathrm{x} \in \Gamma_{\mathrm{z}}:-\lambda_{\mathrm{e}} \frac{\partial \mathrm{T}_{\mathrm{e}}}{\partial \mathrm{n}}=\alpha_{\mathrm{z}}\left[\mathrm{T}_{\mathrm{e}}-\mathrm{T}_{\mathrm{z}}\right] \text { and } \mathrm{x} \in \Gamma_{\mathrm{w}}:-\lambda_{\mathrm{e}} \frac{\partial \mathrm{T}_{\mathrm{e}}}{\partial \mathrm{n}}=\alpha_{\mathrm{w}}\left[\mathrm{T}_{\mathrm{e}}-\mathrm{T}_{\mathrm{w}}\right]
$$

$\mathrm{w}$ - area inside, $\mathrm{z}$-area outside, $\alpha$ - heat transfer coefficient $\partial / \partial \mathrm{n}$ - derivative normal to the edge of the area.

At the contact surface there are continuity conditions $[4,5]$ :

$$
\mathrm{x} \in \Gamma_{\mathrm{e}, \mathrm{è}}:-\lambda_{\mathrm{e}} \frac{\partial \mathrm{T}_{\mathrm{e}}}{\partial \mathrm{n}}=-\lambda_{\grave{\mathrm{e}}} \frac{\partial \mathrm{T}_{\grave{e}}}{\partial \mathrm{n}}, \mathrm{T}_{\mathrm{e}}=\mathrm{T}_{\grave{\mathrm{e}}}
$$

e, è- ideal contact surface, for surface no flux conditions

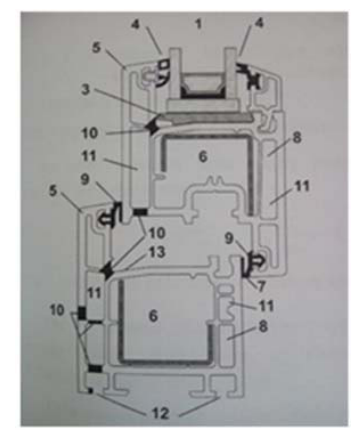

Fig.1. Analysing area: 1-glasses, 3-distance block, 4-gasket, 5-PVC profile, 6- aluminium strengthening, 7-Gasket connector, 8,11- air chamber, 10- drainage hole, 12-wall connector [6].

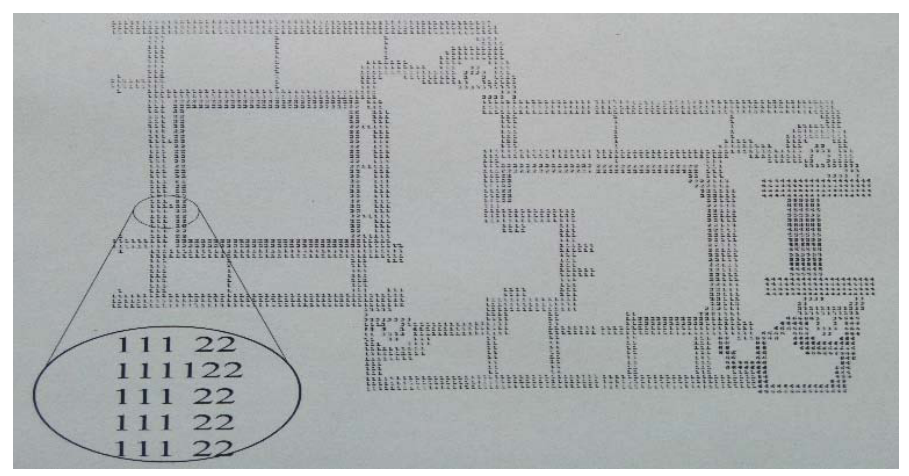

Fig.2. Consideration area and matrix identification [6,13-16]. 


\subsection{Numerical model}

Consider the area was covered with a net differential (Fig. 3).

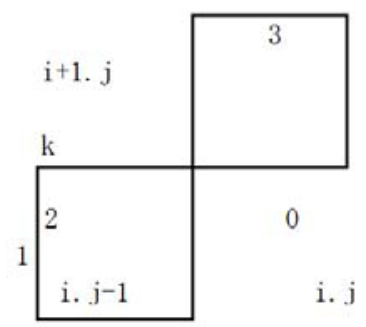

Fig. 3. Five points star net.

Approximation equations shows the relation between central node and neighbor nodes.

$$
\frac{T_{i+1, j}-T_{i, j}}{R_{i+1, j}} \Phi_{i+1, j}+\frac{T_{i-1, j}-T_{i, j}}{R_{i-1, j}} \Phi_{i-1, j}+\frac{T_{i, j-1}-T_{i, j}}{R_{i, j-1}} \Phi_{i, j-1}+\frac{T_{i, j+1}-T_{i, j}}{R_{i, j}+1} \Phi_{i, j+1}=0
$$

R- resistance to heat flow, $\Phi=\frac{1}{h}$.

$$
\begin{array}{r}
A_{0} T_{i, j}-A_{1} T_{i+1, j}-A_{2} T_{i-1, j}-A_{3} T_{i, j-1}-A_{4} T_{i, j+1}=0 \\
T_{i, j}=\frac{A_{1}}{A_{0}} T_{i+1, j}-\frac{A_{2}}{A_{0}} T_{i-1, j}-\frac{A_{3}}{A_{0}} T_{i, j-1}-\frac{A_{4}}{A_{0}} T_{i, j+1}
\end{array}
$$

\subsection{Numerical realization}

The area was covered with net it has steps $\mathrm{h}=0,001 \mathrm{~m}[13,14]$.

Area which was considered has got 10576 points. The matrix type integer (Fig.2).

Window size: S $1465 \mathrm{~mm}$ x H $2195 \mathrm{~mm}$, glass $4 \mathrm{~mm}$, distance between glazing: $12-$ $18 \mathrm{~mm}, \mathrm{~T}_{\text {inside }}=20^{\circ} \mathrm{C}, \mathrm{T}_{\text {outside }}=-20,-15,-10,-5,0$ (in subsequent calculations),$\alpha_{\mathrm{w}}=8.1 \mathrm{~W} / \mathrm{m}^{2} \mathrm{~K}$, $\alpha_{\mathrm{z}}=23 \mathrm{~W} / \mathrm{m}^{2} \mathrm{~K}, \lambda_{\mathrm{sz} 1}=0.744 \mathrm{~W} / \mathrm{mK}, \lambda_{\mathrm{sz} 2}=1.338 \mathrm{~W} / \mathrm{mK}$.

Numerical realization was done used special for this prepared author program.

\section{Results and discussions}

Results simulation show parameters $\mathrm{k}$ and $\mathrm{q}$ for different set points temperatures in term of $-20^{\circ} \mathrm{C}$ to $0^{\circ} \mathrm{C}$. When we take into account $\lambda_{\text {ef }}$ results was increase about $57.5 \%$ what good correlation to real experimental results.

Table 1. Numerical simulation results.

\begin{tabular}{|c|c|c|c|c|}
\hline $\mathrm{N}^{\mathrm{o}}$ & $\mathrm{T}_{\mathrm{z} \text { (outside) }}\left[\mathrm{C}^{0}\right]$ & $\mathrm{T}_{\mathrm{W} \text { (inside) }}\left[\mathrm{C}^{0}\right]$ & $\mathrm{q}\left[\mathrm{W} / \mathrm{m}^{2}\right]$ & $\mathrm{k}\left[\mathrm{W} / \mathrm{m}^{2} \mathrm{~K}\right]$ \\
\hline 1 & -20 & 20 & 48.21 & 1.205 \\
\hline 2 & -10 & 20 & 36.17 & 1.206 \\
\hline 3 & 0 & 20 & 24.12 & 1.206 \\
\hline \multicolumn{5}{|c|}{ When changed for effective conductivity coefficient $\left(\lambda_{\mathrm{ef}}=\lambda_{\text {air }}\right)$} \\
\hline 4 & -20 & 20 & 75.46 & 1.89 \\
\hline 5 & -10 & 20 & 56.60 & 1.89 \\
\hline 6 & 0 & 20 & 37.72 & 1.89 \\
\hline
\end{tabular}

Graphical presentation simulation which result show in Table 1 (Author program). 


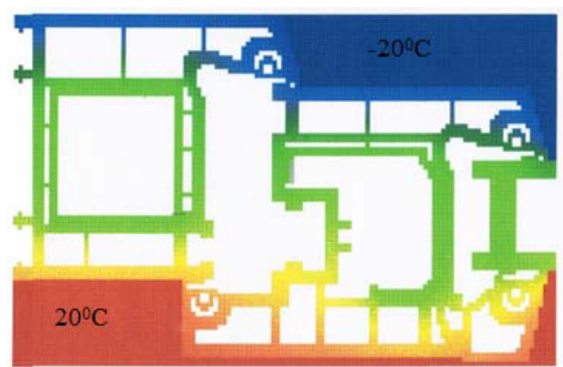

Fig. 4. Window set: $-20^{\circ} \mathrm{C}$ outside $20^{\circ} \mathrm{C}$ inside.

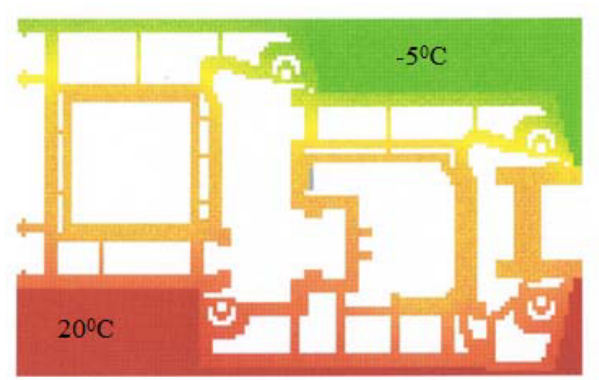

Fig. 6. Window set: $-5^{0} \mathrm{C}$ outside $20^{\circ} \mathrm{C}$ inside.

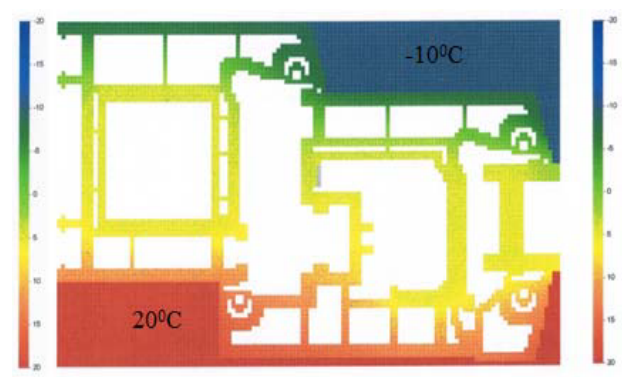

Fig. 5. Window set: $-10^{\circ} \mathrm{C}$ outside $20^{\circ} \mathrm{C}$ inside.

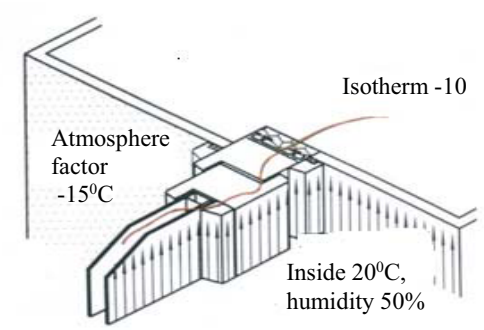

Fig. 7. Isotherm $-10^{\circ} \mathrm{C}$ inside panel [1].

Graphical presentation show areas when different colours are assigned to a specific temperature. Area in specific colour show distribution temperature for interpretation when we have place which must be structural remodel.

Table 2. Show termoisolation optimum distance between glasses, size affected heat transfer coefficient and gases have got changes for result [7].

\begin{tabular}{|c|c|c|c|c|c|c|c|}
\hline$N^{\circ}$ & $\begin{array}{c}\text { Distance between } \\
\text { glasses }[\mathrm{mm}]\end{array}$ & $\begin{array}{c}\text { Panel } \\
\mathrm{H}[\mathrm{mm}]\end{array}$ & gas & $\begin{array}{c}\mathrm{T} \\
\text { outside[ }^{\circ} \\
\mathrm{C}]\end{array}$ & glass & $\begin{array}{c}\text { Heat } \\
\text { lost[J] }\end{array}$ & $\mathrm{k}\left[\mathrm{W} / \mathrm{m}^{2} \mathrm{~K}\right]$ \\
\hline 1. & 15 & 1400 & Air & -20 & quartz & 92 & 1.64 \\
\hline 2. & 15 & 1400 & Argon & -20 & float & 67.7 & 1.21 \\
\hline 3. & 15 & 1400 & Air & -20 & float & 83 & 1.48 \\
\hline 4. & 15 & 1400 & Air & -10 & float & 61.4 & 1.46 \\
\hline 5. & 15 & 1400 & Air & 0 & float & 37.5 & 1.34 \\
\hline 6. & 18 & 1400 & Air & -20 & float & 83.2 & 1.49 \\
\hline 7. & 12 & 1400 & Air & -20 & float & 90.2 & 1.61 \\
\hline 3 & 15 & 1400 & Air & -20 & float & 83 & 1.48 \\
\hline 8. & 15 & 2600 & Air & -20 & float & 154.1 & 1.48 \\
\hline
\end{tabular}

In table 2 we have results part of interesting simulations which shows optimal distance between glasses $(15 \mathrm{~mm})$ which guarantee maximum isolation. When we increases or decreases the distance between glasses, $\mathrm{k}$ changes what means heat transfer have to high level and we lost optimum termoisolation. Similar situation we have when we use quartz glass not float glass. Heat transfer coefficient increase about $11 \%$ when we have in windows quartz glass. If we use different gasses not air, example Argon or Krypton heat transfer coefficient ensure better termoisolation about $22 \%$. When we would like the same 
result when we used Argon or Krypton we must between glasses used "air vacuum".

Interesting situation we have when we used higher window panel and compare to standard panel.

Heat transfer coefficient it is constant. Meaning highest panel set not decrease termoisolation.

We were check different construction as well for find better opportunity. Double glasses window which have between glasses on the middle spectroselective foil (in PVD technology was sputtered metal oxides). Results: $\mathrm{k}=1 \mathrm{~W} / \mathrm{m}^{2} \mathrm{~K}$. Radiation $\mathrm{UV}=0$. Short IR only $6 \%$. Because this foil stopped radiation longer than $380 \mathrm{~nm}$ flowers don't have light to life!

As well shadow coefficient increased to 0.66 , resulting for this we have better protection against overheating. Visibility $88 \%$.

Graphical presentation simulation which result show in Table 2.

Convection which we see on the Fig. 8. and Fig. 10 was simulation done in commercial program fluent 5 shows Bernard cells. Fig. 8, 9, 11,12 shows graphical presentation results from Table 2 .

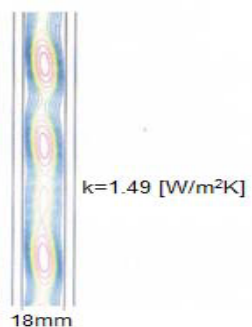

Fig. 8. Set of glass for optimality distance between glazed [8].

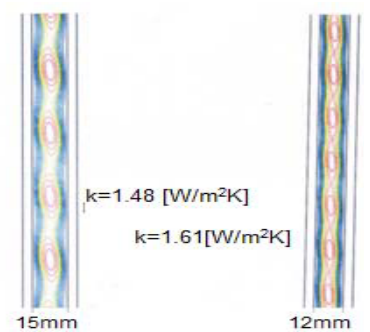

$$
12 \mathrm{~mm}
$$

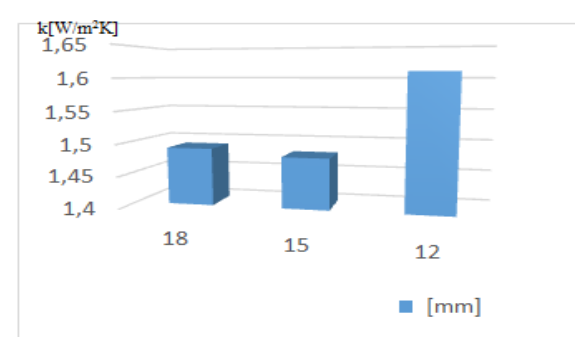

Fig. 9. Optimum distance between glasses.

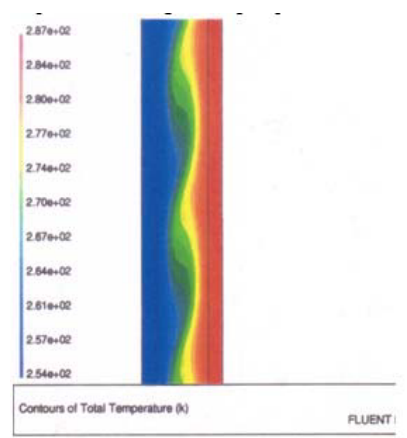

Fig. 10. We have Bernard cells characteristic for free convection in close space between glasses. [7, 9].

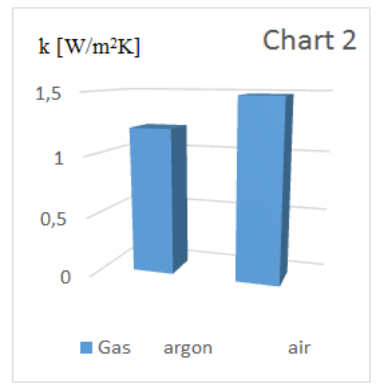

Fig. 11. Affect heat transfer coefficient in glass panel used different gasses [10].

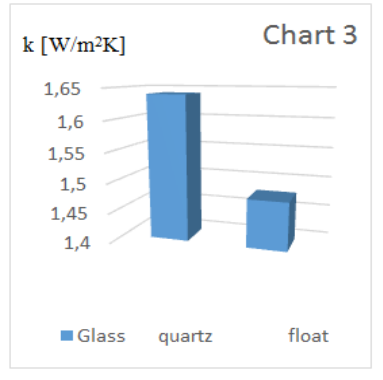

Fig. 12. Affect heat transfer coefficient in glass panel used different glass [10].

\section{Experimental study}

Results measured in heating chamber have a very good correlation with simulation results. Experimental verification helped confirm the correctness of the tests simulation.

Differences of $3 \%$ between the method of calculation and experimental confirms that the application of the calculation method is justified in order to search for the optimal 
design solution for partitions heat.

Windows samples were tested in a heating chamber [13].

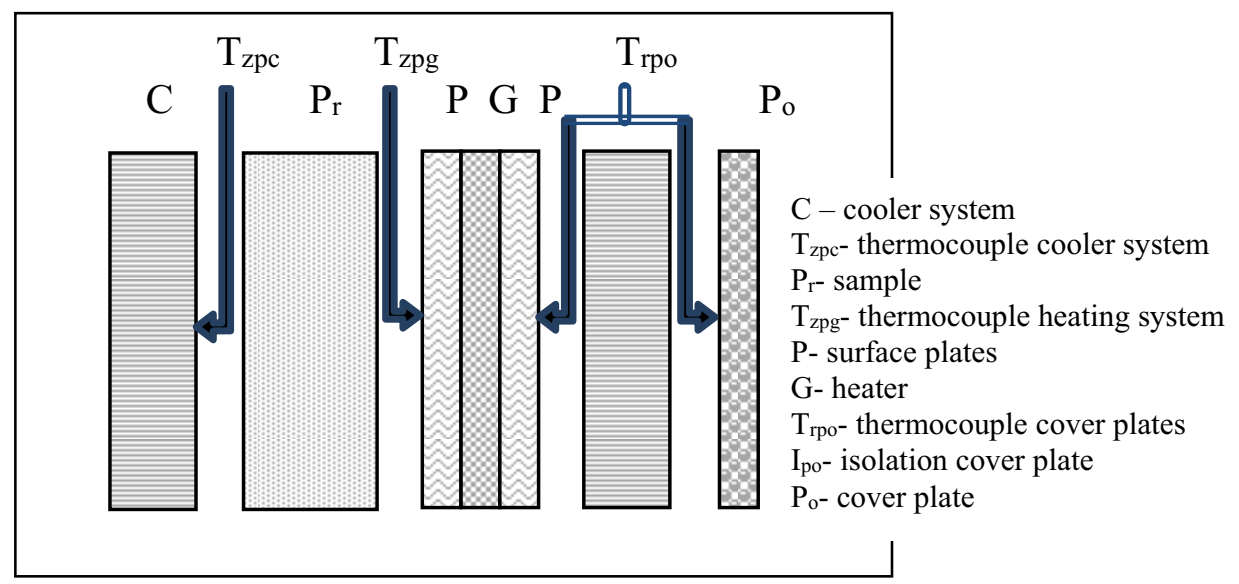

Fig. 13. Heating chamber scheme $[11,12]$.

Parameters for samples: $\mathrm{T}_{1}=20^{\circ} \mathrm{C}, \mathrm{T}_{2}=-20^{\circ} \mathrm{C}, \mathrm{h}_{\mathrm{e}}=23 \mathrm{~W} / \mathrm{m}^{2} \mathrm{~K}, \mathrm{~h}_{\mathrm{i}}=8 \mathrm{~W} / \mathrm{m}^{2} \mathrm{~K}$

Table 3. Results measurements in heating chamber.

\begin{tabular}{|c|c|c|}
\hline Set of glass in panel & Gas between set of glasses & $\begin{array}{c}\mathrm{k} \\
{\left[\mathrm{W} / \mathrm{m}^{2} \mathrm{~K}\right]}\end{array}$ \\
\hline $4-15-4$ float & air & 1.49 \\
\hline $4-15-4$ quartz & air & 1.67 \\
\hline $4-15-4$ float & air & 1.62 \\
\hline $4-15-4$ float & air & 1.51 \\
\hline $4-15-4$ float & argon & 1.23 \\
\hline
\end{tabular}

\section{Conclusions}

1. Between both methods we have a good correlation simulation where for set 4-15-4 float was $\mathrm{k}=1.48 \mathrm{~W} / \mathrm{m}^{2} \mathrm{~K}$, used argon $\mathrm{k}=1.21 \mathrm{~W} / \mathrm{m}^{2} \mathrm{~K}$ and for quartz $\mathrm{k}=1.64 \mathrm{~W} / \mathrm{m}^{2} \mathrm{~K}$ and experimental where was 4-15-4 float was $\mathrm{k}=1.52 \mathrm{~W} / \mathrm{m}^{2} \mathrm{~K}$, used argon $\mathrm{k}=1.23 \mathrm{~W} / \mathrm{m}^{2} \mathrm{~K}$ and for quartz $\mathrm{k}=1.67 \mathrm{~W} / \mathrm{m}^{2} \mathrm{~K}$ means only $3 \%$ difference, this allow the use of simulation methods in research application.

2. We found optimum distance between glasses in window panel for the best isolation, it is $15 \mathrm{~mm}$ between glasses in window panel. If we try increase distance between glasses slowly increases $\mathrm{k}$ (from $\mathrm{k}=1.48 \mathrm{~W} / \mathrm{m}^{2} \mathrm{~K}$ for $15 \mathrm{~mm}$ to $\mathrm{k}=1.49 \mathrm{~W} / \mathrm{m}^{2} \mathrm{~K}$ for $18 \mathrm{~mm}$ ), if we try decrease distance much faster changed $\mathrm{k}$ (from $\mathrm{k}=1.48 \mathrm{~W} / \mathrm{m}^{2} \mathrm{~K}$ for $15 \mathrm{~mm}$ to $\mathrm{k}=1.61 \mathrm{~W} / \mathrm{m}^{2} \mathrm{~K}$ for $12 \mathrm{~mm}$ )

3. Used different gasses between glasses changed termoisolations in set of window. Argon changed approximately $26 \%$ heat transfer coefficient from $\mathrm{k}=1.48 \mathrm{~W} / \mathrm{m}^{2} \mathrm{~K}$ (air) tok $=1.21$ $\mathrm{W} / \mathrm{m}^{2} \mathrm{~K}$ (argon)

4. Different kind of glass affect heat transfer coefficient too. Set 4-15-4 float glass with air 
$\mathrm{k}=1.48 \mathrm{~W} / \mathrm{m}^{2} \mathrm{~K}$ but the same set with air for quartz glass $\mathrm{k}=1.64 \mathrm{~W} / \mathrm{m}^{2} \mathrm{~K}$ this is different more than $9 \%$.

5. Possibility used electric wire to heat up inside glass for better isolation and reduce heater bridge on the button for considered size window $160 \mathrm{~W}-300 \mathrm{~W}$ according to temperature outside building.

6. Alternative connection to solar panel on sun water panel to heat up.

7. Possibility connect to geothermal heat pomp for heat up frame and glass or in summer cool down to $12.2^{\circ} \mathrm{C}$ and will be works like small air-condition.

\section{References}

1. B. Mochnacki, J.S. Suchy, Numerical methods in computations of foundry processes, (PFTA, Cracow, 1995)

2. D. Andrzejewski, Mechanika 263, 63 (2000)

3. J. S. Suchy, D. Andrzejewski and others, State Committee for Scientific Research, Research project no. PC-2511

4. S. J. Gdula, R. Bialecki, K. Kurpisz, A.J. Nowak, A. Sucheta, Heat conduction (PWN, Warszawa, 1984)

5. E. Kostowski, series CMP, 97 (1988)

6. Information from KBE Sp .z o.o. Wroclaw

7. Fluent Manual, Heat Transfer and Numerical Algorithms. Fluent Software (MTG-96173, Fluent Europe Ltd.)

8. D.K. Edwards, Heat transfer 12, 4 (1976)

9. D. Andrzejewski, Mechanika 279, 72 (2001)

10. Andrzejewski D., Laboratory tests - Protection against noise and reducing energy needs through the use of modern joinery, III Forum Innovation and Technology Transfer (Opole, 2001)

11. Polish Committee for Standardization, Building components and building elementsthermal resistance and heat transfer coefficient PN-EN ISO 6946: 1998

12. Polish Committee for Standardization, Determination of the coefficient of heat transfer U PN-EN 674:1999

13. J. C. Cavendish, D. A. Field, W.H. Frey, Automatic Mesh Generation: a Finite Element/ Computer aided geometric interface. mathematics of Finite Elements and Applications, Mafelap V (Academic press, 1984)

14. Y. Depeursinge, Interactive Generation of Three dimensional Meshes., In: Mathematics of Finite elements and Applications Mafelap IV (Academic Press, 1981)

15. J. Haüser, C. Taylor, Numerical grid generation in Computational Fluid dynamics (Pineridge Press, Swansea, 1986)

16. O.C. Zientkiewicz, The Finite Element Method (McGraw, London, 1977) 